JOURNAL OF

SYMPLECTIC GEOMETRY

Volume 7, Number 3, 311-335, 2009

\title{
COHOMOLOGY OF COURANT ALGEBROIDS WITH SPLIT BASE
}

\author{
Grégory Ginot and Melchior Grützmann
}

In this paper we study the cohomology $H_{\mathrm{st}}^{\bullet}(E)$ of a Courant algebroid $E$. We prove that if $E$ is transitive, $H_{\mathrm{st}}^{\bullet}(E)$ coincides with the naive cohomology $H_{\text {naive }}^{\bullet}(E)$ of $E$ as conjectured by Stiénon and Xu. For general Courant algebroids $E$ we define a spectral sequence converging to $H_{\mathrm{st}}^{\bullet}(E)$. If $E$ is with split base, we prove that there exists a natural transgression homomorphism $T_{3}$ (with image in $H_{\text {naive }}^{3}(E)$ ) which, together with $H_{\text {naive }}^{\bullet}(E)$, gives all $H_{\mathrm{st}}^{\bullet}(E)$. For generalized exact Courant algebroids, we give an explicit formula for $T_{3}$ depending only on the Ševera characteristic clas of $E$.

\section{Introduction}

The purpose of this paper is to study the cohomology of Courant algebroids. The Courant bracket was first introduced by Courant in 1990 (see [Cou90]) in order to describe Dirac manifolds, a generalization of presymplectic and Poisson manifolds. In $1997 \mathrm{Liu}$, Weinstein and $\mathrm{Xu}$ introduced the notion of a Courant algebroid in order to describe Manin triples for Lie bialgebroids ([LWX97]). Recently, Courant algebroids have been used as a background to describe generalized complex geometry, see [Hit03, Gua04] and as target spaces for three-dimensional topological field theory [Ike01, Ike03, Par01, HP04, Roy07].

Roughly speaking, a Courant algebroid is a pseudo-Euclidean vector bundle $E \rightarrow M$ together with an anchor map $\rho: E \rightarrow T M$ and a bracket [.,.] on $\Gamma E$ which satisfy the basic identities, e.g., skew-symmetry, Jacobi identity, Leibniz rule and ad-invariance, only up to anomalies (which are exact terms). Up to the anomalies, the bracket and the anchor map are similar to those of a Lie algebroid. Indeed, Courant algebroids appear to be the right framework for pseudo-metric vector bundles equipped with something like a 
quadratic Lie algebra structure. A primary example of a Courant algebroid is given by the double $A \oplus A^{*}$ of a Lie bialgebroid $A$ or a Lie quasi-bialgebroid, that is a Lie bialgebroid twisted by a 3 -form [Roy99].

An important step forward by Roytenberg was a description of Courant algebroids in terms of a derived bracket as introduced by KosmannSchwarzbach in [KS96], see [Roy01], or equivalently in terms of a nilpotent odd operator (also known as $Q$-structure). Hence the Courant algebroid structure with its intricate axioms can all be encoded in a cubic function $H$ on a graded symplectic manifold and its derived bracket. To do so, one goes into the context of graded manifolds and considers the graded manifold $E[1]$. The pseudo-metric on $E$ makes $E[1]$ into a (graded) Poisson manifold.

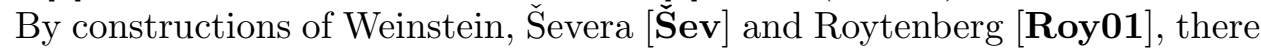
is a minimal symplectic realization $(\mathcal{E},\{.,\}$.$) of E[1]$. Now, there is a cubic Hamiltonian $H$ satisfying $\{H, H\}=0$ on $\mathcal{E}$ encoding the Courant algebroid structure together with the symplectic structure of $\mathcal{E}$. For instance, the Courant bracket is given by the formula $[\phi, \psi]=\{\{H, \phi\}, \psi\}$.

The derived bracket construction also leads to a natural notion of cohomology of a Courant algebroid. Since $\{H, H\}=0$, the operator $Q=\{H,$.$\} :$ $\mathcal{C}^{\infty}(\mathcal{E}) \rightarrow \mathcal{C}^{\infty}(\mathcal{E})$ is a differential. Hence one can define the cohomology of $E$ as the cohomology $H^{\bullet}\left(\mathcal{C}^{\infty}(\mathcal{E}), Q\right)$. So far, there are only few examples of Courant algebroids for which the cohomology is known. For instance when $E \cong T^{*} M \oplus T M$ is an exact Courant algebroid, its cohomology is isomorphic to the de Rham cohomology of $M$. On the other hand, if the base is a point, $E$ is a Lie algebra (together with an ad-invariant pseudo-metric) and its cohomology is isomorphic to its cohomology as a Lie algebra. One reason which makes $H^{\bullet}\left(\mathcal{C}^{\infty}(\mathcal{E}), Q\right)$ rather difficult to treat is that its construction relies on the minimal symplectic realization $\mathcal{E}$ and not just on $E$ or $E^{*}$ itself. In particular, it is quite different from the usual cohomology theories for "Lie theoretic objects" such as Lie algebroids or Leibniz algebras where the cohomology is defined using a differential given by a Cartan-type formula. For instance, the (de Rham) cohomology of a Lie algebroid $(A,[.,],. \rho: A \rightarrow M)$ over $M$ is the cohomology of the complex of forms $\left(\Gamma\left(\Lambda^{\bullet} A^{*}\right), d_{A}\right)$, where the differential $d_{A}$ is given, for $\alpha$ an $n$-form and $\psi_{1}, \ldots, \psi_{n} \in \Gamma(A)$, by the Cartan formula

$$
\begin{aligned}
\left\langle d_{A} \alpha, \psi_{1} \wedge \ldots \wedge \psi_{n+1}\right\rangle:= & \sum_{i=1}^{n+1}(-1)^{i+1} \rho\left(\psi_{i}\right)\left\langle\alpha, \psi_{1} \wedge \ldots \wedge \widehat{\psi}_{i} \wedge \ldots \wedge \psi_{n}\right\rangle \\
& +\sum_{i<j}(-1)^{i+j}\left\langle\alpha,\left[\psi_{i}, \psi_{j}\right] \wedge \psi_{1} \wedge \ldots \wedge \widehat{\psi}_{i} \ldots \widehat{\psi_{j}} \ldots \psi_{n}\right\rangle .
\end{aligned}
$$

This formula does not make sense for a Courant algebroid $E$ in place of $A$ since, there is no definition of a Courant algebroid for which the bracket 
can be made skew-symmetric and the Jacobi identity and the Leibniz rule are strictly satisfied. Nevertheless, Stiénon-Xu [SX08] recently observed that formula (1.1) makes sense if one restricts $E$ to the kernel ker $\rho$ of its anchor map. Precisely, they proved that formula (1.1) defines a differential $d_{\text {naive }}$ on $\Gamma\left(\Lambda^{\bullet} \operatorname{ker} \rho\right)$, where the pairing $\langle.,$.$\rangle in the formula is induced by the$ identification of $E$ and $E^{*}$ by the pseudo-metric. They call the cohomology of $\left(\Gamma\left(\Lambda^{\bullet} \operatorname{ker} \rho\right), d_{\text {naive }}\right)$ the naive cohomology of $E$ and denoted it $H_{\text {naive }}^{\bullet}(E)$. Furthermore, they conjectured that for a transitive Courant algebroid $E$, i.e. a Courant algebroid with surjective anchor map, the naive cohomology of $E$ is isomorphic to the (standard) cohomology $H^{\bullet}\left(\mathcal{C}^{\infty}(\mathcal{E}), Q\right)$ of $E$. We prove this conjecture in Section 4.2, see Corollary 4.10. A nice feature of the naive cohomology is that it can be calculated using the same techniques as for Lie algebroids since it is defined similarly.

Another goal of this paper is to study the cohomology of general Courant algebroids. We show that, in general, the naive cohomology and the standard cohomology are related by a spectral sequence. Spectral sequences are a useful tool in geometry and topology. For instance, given a map $X \stackrel{\pi}{\rightarrow} Y$, the Leray spectral sequence allows one to compute the cohomology of $X$ in terms of the (sheaf) cohomology of $Y$ and the inverse images under $\pi$. This spectral sequence can be very complicated if $\pi$ is not nice enough. On the other hand, it takes a much simpler form, when $\pi$ is a fibration with a trivial action of $\pi_{1}(Y)$ on the fiber $F$, relating the cohomology of $X$ to the cohomology of $Y$ and $F$. Spectral sequence also leads to transgression [BT82, McC85]. Our spectral sequence can be thought of as some sort of an analogue of the Leray spectral sequence of the morphism of graded manifolds $\mathcal{E} \rightarrow(\operatorname{ker} \rho)^{*}[1]$ induced by the pseudo-metric and the map $\mathcal{E} \rightarrow E[1]$. Indeed, the pseudometric allows to see sections of $\Gamma(\operatorname{ker} \rho)$, which are functions on $(\operatorname{ker} \rho)^{*}[1]$, as functions on $E[1]$ which can be pulled back to functions on $\mathcal{E}$. Then, the ideal $I=(\Gamma(\operatorname{ker} \rho[1])) \subset \mathcal{C}^{\infty}(\mathcal{E})$ is a differential ideal which induces a filtration on the complex $\left(\mathcal{C}^{\infty}(\mathcal{E}), Q\right)$. The filtration gives rise to a spectral sequence converging to $H^{\bullet}\left(\mathcal{C}^{\infty}(\mathcal{E}), Q\right)$ that we call the naive ideal spectral sequence, see Section 4.1. Note that if $X \stackrel{\pi}{\rightarrow} Y$ is a fibration of manifolds, the Leray spectral sequence in de Rham cohomology is obtained by a similar construction using the filtration induced on the differential algebra of forms $\Omega^{\bullet}(X)$ by the (differential) ideal generated by (the pullback along $\pi$ of) $\Omega^{1}(Y)$. Also note that in terms of super geometry $\Omega^{\bullet}(X)$ is the algebra of smooth functions on $T[1] X$.

The next important part of this paper is to compute explicitly the naive ideal spectral sequence in terms of smooth geometric data. This computation involves taking quotient of $T M$ by $D=\operatorname{im} \rho$, thus, one has to assume this quotient is nice. In Section 4.2, we consider the case of Courant algebroids with split base, i.e., Courant algebroids for which the base is isomorphic to a product $L \times N$ of manifolds and $D=\operatorname{im~} \rho \cong T L \times N$. Note that 
any transitive Courant algebroid is with split base. In the general case of split base, the naive cohomology is an approximation of the cohomology of $E$ in the sense that the term $E_{2}^{\bullet \bullet \bullet}$ of the spectral sequence is the tensor product of the naive cohomology with symmetric multivector fields on $N$, see Proposition 4.8 .

Furthermore, by transgression, the spectral sequence induces a map $T_{3}: \mathcal{X}(N) \rightarrow H_{\text {naive }}^{3}(E)$ that we call the transgression homomorphism (here $\mathcal{X}(N)$ is the space of vector fields on $N)$. This map is a cohomological characteristic class. Indeed, we show that the naive cohomology and the transgression homomorphism determines explicitly the (standard) cohomology of $E$, see Theorem 4.12 .

There is a nice class of Courant algebroids parametrized by closed 3-forms. According to Ševera [ک̌́ev99], exact Courant algebroids over $M$, i.e., Courant algebroids fitting in an exact sequence $0 \rightarrow T^{*} M \rightarrow E \rightarrow T M \rightarrow 0$ are in bijection with cohomology classes of closed 3-forms on $M$ [Г̌W01, Roy99]. We define a generalized exact Courant algebroid to be a regular Courant algebroid fitting into an exact sequence $0 \rightarrow D^{*} \rightarrow E \rightarrow D \rightarrow 0$ of bundles over $M$, where $D=\operatorname{im} \rho$. By Ševera's argument, we get that generalized exact Courant algebroids are in bijection with cohomology classes of closed 3-forms on (the Lie algebroid) $D$. The class associated to a generalized exact Courant algebroid is called its Ševera characteristic class. If, furthermore, $E$ is with split base $D \cong T L \times N$, then a 3 -form on $D$ lies in $\Omega^{3}(L) \otimes \mathcal{C}^{\infty}(N)$. We prove that for a generalized exact Courant algebroid with split base, the transgression homomorphism is given by the map $\mathcal{X}(N) \ni X \mapsto(1 \otimes X)(C) \in H^{3}(L) \otimes \mathcal{C}^{\infty}(N) \cong H_{\text {naive }}^{3}(E)$ where $[C]$ is the Severa characteristic class, see Proposition 5.6. As a consequence, we obtain an explicit computation of the cohomology of $E$ in terms of $[C]$, see Corollary 5.7.

The plan of the paper is as follows. In Section 2, we recall the definition of Courant algebroids and their cohomology, following [Roy99, Roy01]. In Section 3, we explain the definition of the naive cohomology following [SX08]. We define the naive ideal spectral sequence in Section 4.1. Then we compute the spectral sequence in the case of Courant algebroids with split base in Section 4.2. In particular, we prove the Conjecture of Stiénon-Xu, see Corollary 4.10. We also define the transgression homomorphism. The second main result in this section is Theorem 4.12 computing the cohomology of a Courant algebroid with split base. In section 5, we study generalized exact Courant algebroids, defining their Ševera characteristic class and giving their classification, Proposition 5.3. We then compute the transgression homomorphism in terms of the characteristic class, see Proposition 5.6. For the reader's convenience we included an appendix recalling the basic facts and definition of graded (and super) geometry that we need. 


\section{Courant algebroids and their cohomology}

In this section we recall the notion of Courant algebroids and their cohomology. We work in the context of graded manifolds as described in Appendix 6 . We refer to [Vor91, Vor02, Šev05, Roy01] for more details.

The Courant bracket was originally defined as a skew-symmetric bracket satisfying the Jacobi identity and Leibniz rule only up to some anomalies [Cou90]. As was shown by Roytenberg in his thesis [Roy99], there is an alternative equivalent formulation of Courant algebroids, namely one with a bracket fulfilling a certain form of the Jacobi identity (in fact the Leibniz rule for the bracket see axiom (1) in Definition 2.1) but no longer skew-symmetric originally due to Dorfman [Dor87, Dor93]. We recall the latter definition since it is more convenient in our context and also the more closely related to Loday algebroids as in [SX08].

Definition 2.1 (Courant algebroid). A Courant algebroid is a vector bundle $E \rightarrow M$ equipped with a symmetric non-degenerate bilinear product $\langle.,$.$\rangle :$ $E \otimes_{M} E \rightarrow \mathbb{R}$, an $\mathbb{R}$-linear bracket on the sections of $E$ and a bundle map $\rho: E \rightarrow T M$, called the anchor map. These three operations have to satisfy to the following rules:

$$
\begin{aligned}
{\left[\phi,\left[\psi_{1}, \psi_{2}\right]\right] } & =\left[\left[\phi, \psi_{1}\right], \psi_{2}\right]+\left[\psi_{1},\left[\phi, \psi_{2}\right]\right], \\
{[\phi, f \psi] } & =\rho(\phi) f \cdot \psi+f[\phi, \psi], \\
{[\phi, \phi] } & =\frac{1}{2} \rho^{*} d\langle\phi, \phi\rangle, \\
\rho(\phi)\langle\psi, \psi\rangle & =2\langle[\phi, \psi], \psi\rangle,
\end{aligned}
$$

where $\phi, \psi, \psi_{1}, \psi_{2}$ are sections into $E$ and $f$ is a smooth function on $M$.

Remark 2.2. Note that the bracket [.,.] is not skew-symmetric. Also, some authors denote this bracket $\circ$, for instance see [SX08, Roy01].

Remark 2.3. Since the bilinear form $\langle.,$.$\rangle is non-degenerate, a Courant alge-$ broid is a pseudo-Euclidean vector bundle. In particular, there is a canonical identification of $E$ and $E^{*}$ induced by the pseudo-metric.

A Courant algebroid is called transitive if the anchor map $\rho: E \rightarrow T M$ is surjective. It is called regular if $\rho$ is of constant rank.

Due to an observation of Uchino ([Uch02]), the Jacobi identity (2.1) and Leibniz rule (2.2) imply that the anchor map preserves the bracket: for all $\psi_{1}, \psi_{2} \in \Gamma(E)$, one has

$$
\rho\left[\psi_{1}, \psi_{2}\right]=\left[\rho \psi_{1}, \rho \psi_{2}\right]_{T M}
$$

Therefore, in the regular case the image of the anchor map is a Lie algebroid.

The Courant bracket can also be obtained as a derived bracket [KS96, Roy01] on a (degree 2) graded symplectic manifold. More precisely, the pseudo-metric on $E$ induces a structure of (degree 2) graded Poisson 
manifold on $E[1]$. There is a minimal symplectic realization $\mathcal{E}$ of $(E[1],\langle.,\rangle$. constructed as follows. When $E$ is a split vector bundle $E=A \oplus A^{*}$ (with the obvious pairing), $\mathcal{E}$ is just $T^{*}[2] A[1]$ with the usual graded symplectic structure (see Appendix 6). In the general case (where $\langle.,$.$\rangle might not even have$ split signature), a construction of $\mathcal{E}$ was found by Weinstein, Ševera [Šev] and Roytenberg [Roy01]. First consider the graded symplectic manifold $T^{*}[2] E[1]$. Let $\imath: E[1] \rightarrow E \oplus E^{*}[1]$ be the isometric embedding (given by the pseudo-metric). The minimal symplectic realization of $E[1]$ is the pullback $\mathcal{E}:=\imath^{*} T^{*}[2] E[1]=E[1] \times_{E \oplus E^{*}[1]} T^{*}[2] E[1]$ of $T^{*}[2] E[1]$ along $\imath$. We denote $\pi: \mathcal{E} \rightarrow E[1]$ the canonical bundle projection. Note that $\mathcal{E}$ fits into the following short exact sequence of graded fiber-bundles over $M$ :

$$
0 \rightarrow T^{*}[2] M \rightarrow \mathcal{E} \stackrel{\pi}{\rightarrow} E[1] \rightarrow 0 .
$$

Roytenberg [Roy01] proved that there is a cubic Hamiltonian $H$ on $\mathcal{E}$ encoding the Courant algebroid structure on E. More precisely, the Hamiltonian $H$ satisfies the following properties:

(1) The Courant bracket is given by the derived bracket $[\phi, \psi]=$ $\{\{H, \phi\}, \psi\}$ where we identify sections $\phi, \psi$ of $E$ with fiber-linear functions on $E$ by the pseudo-metric $\langle.,.\rangle{ }^{1}$

(2) The anchor map is given, for any $\psi \in E$, by the formula $\rho(\psi)=$ $\{\{\psi, H\},$.$\} .$

(3) $H$ is nilpotent, i.e., $\{H, H\}=0$.

Remark 2.4. Note that axioms $(2.2-2.4)$ of a Courant algebroid (in Definition 2.1) now follow directly from the derived bracket construction. The first axiom, i.e., the Jacobi identity is equivalent to $\{H, H\}=0$.

The derived bracket approach allows us to define a natural notion of cohomology for Courant algebroids. The Hamiltonian $H$ gives a degree 1 derivation $Q=\{H,-\}$ which is of square zero (since $\{H, H\}=0$ ). Furthermore, $Q$ maps graded functions on $\mathcal{E}$ to graded functions on $\mathcal{E}$. We denote $A^{\bullet}:=C^{\infty}(\mathcal{E})$ the graded functions on the minimal symplectic realization $\mathcal{E} \stackrel{\pi}{\rightarrow} E$ of $E$, where, by definition $A^{n}$ stands for the functions of degree $n$. Then $\left(A^{\bullet}, Q\right)$ is a complex.

Therefore, the following definition due to Roytenberg [Roy01] makes sense.

Definition 2.5 (Cohomology of Courant algebroids). Let $(E \rightarrow M,[.,],. \rho$, $\langle.,\rangle$.$) be a Courant algebroid. Let H$ be a function of degree 3 on the symplectic realization $\mathcal{E}$ generating the Courant bracket. The cohomology of the Courant algebroid $E$ is the cohomology of the complex $\left(A^{\bullet}=C^{\infty}(\mathcal{E}), Q\right)$ equipped with the differential $Q:=\{H,$.$\} .$

\footnotetext{
${ }^{1}$ The derived brackets go back to an idea of Kosmann-Schwarzbach [KS96]
} 
We denote by $H_{\text {std }}^{\bullet}(E)$ the above cohomology of the Courant algebroid $E$. Note that $\left(A^{\bullet}, Q\right)$ is a differential graded commutative algebra, thus $H_{\text {std }}^{\bullet}(E)$ is a graded commutative algebra.

Let us further introduce coordinates on $\mathcal{E}$ for later use. We denote $x^{i}$ the coordinates on the base $M, p_{i}$ their conjugates of degree 2 and $\xi^{a}$ the (pseudo) orthogonal fiber-coordinates of degree 1 on $E$. Then the cubic Hamiltonian reads in coordinates as

$$
H=\rho_{a}^{i}(x) p_{i} \xi^{a}+\frac{1}{6} C_{a b c}(x) \xi^{a} \xi^{b} \xi^{c},
$$

where $\rho\left(\xi_{a}\right)=\rho_{a}^{i}(x) \frac{\partial}{\partial x^{i}}$ encodes the anchor map and $C_{a b c}:=\left\langle\left[\xi_{a}, \xi_{b}\right], \xi_{c}\right\rangle$ are the structure functions of the bracket.

\section{Naive cohomology}

In this section, we recall the definition of the naive cohomology of a Courant algebroid [SX08]. It is less involved than Definition 2.5; for instance, it does not use a symplectic realization of $E$.

Mimicking the definition of the differential giving rise to the cohomology of Lie algebroids, the idea is to consider an operator $d: \Gamma\left(\Lambda^{\bullet} E\right) \rightarrow \Gamma\left(\Lambda^{\bullet+1} E\right)$ given by the Cartan formula

$$
\begin{aligned}
\left\langle d \alpha, \psi_{1} \wedge \ldots \wedge \psi_{n+1}\right\rangle:= & \sum_{i=1}^{n+1}(-1)^{i+1} \rho\left(\psi_{i}\right)\left\langle\alpha, \psi_{1} \wedge \ldots \wedge \widehat{\psi}_{i} \wedge \ldots \wedge \psi_{n+1}\right\rangle \\
& +\sum_{i<j}(-1)^{i+j}\left\langle\alpha,\left[\psi_{i}, \psi_{j}\right] \wedge \psi_{1} \wedge \ldots \wedge \widehat{\psi}_{i} \ldots \widehat{\psi_{j}} \ldots \psi_{n+1}\right\rangle
\end{aligned}
$$

where $\psi_{1}, \ldots \psi_{n+1}$ are sections of $E$ and $\alpha \in \Gamma\left(\Lambda^{n} E\right)$ is identified with an $n$-form on $E$, i.e., a section of $\Lambda^{n} E^{*}$ by the pseudo-metric. However, the formula (3.1) is not well defined because it is not $\mathcal{C}^{\infty}(M)$-linear in the $\psi_{i}$ (due, for instance, to axioms 2.2 and 2.3 in Definition 2.1. The operator $d$ does not square to zero either since it is not skewsymmetric in the $\psi_{i}{ }^{2}$ Nevertheless, Stiénon-Xu [SX08] noticed that formula (3.1) for $d$ becomes $\mathcal{C}^{\infty}(M)$-linear in the $\psi_{i}$ when one restricts to $\alpha \in \Gamma\left(\Lambda^{n}(\operatorname{ker} \rho)\right) .^{3}$ In fact they proved the following

Lemma 3.1. Formula (3.1) yields a well-defined operator $d: \Gamma\left(\Lambda^{\bullet} \operatorname{ker} \rho\right) \rightarrow$ $\Gamma\left(\Lambda^{\bullet+1}\right.$ ker $\left.\rho\right)$. Moreover, one has $d \circ d=0$.

\footnotetext{
${ }^{2}$ Using the skew-symmetric bracket does not help either, because it only fulfills a modified Jacobi identity.

${ }^{3}$ In this case using the skewsymmetric or Jacobi-fulfilling bracket does not matter since the difference is exact, thus vanishes in the inner product with ker $\rho$.
} 
Note that ker $\rho$ may be a singular vector bundle. ${ }^{4}$

Proof. The first claim follows from the fact that the failure of the Leibniz rule on the left-hand side is an exact term, i.e., is in the image of $\rho^{*}$ and that $\rho \circ \rho^{*}=0$. Now the terms for $d \circ d$ add up to zero using the Jacobi identity as in the Lie algebroid case, since all terms are equivalent to those using the skew-symmetric bracket. See [SX08] Section 1 for more details.

Remark 3.2. For a Lie algebroid $A$, the space $\left(\Gamma\left(\Lambda^{\bullet} A^{*}\right), d\right)$, where $d$ is the operator given by formula (3.1), defines its cohomology. In particular, it calculates the de Rham cohomology of $M$ when $A=T M$.

Thanks to the pseudo-metric $\langle.,$.$\rangle on E$, one can view $\Gamma\left(\Lambda^{n} E\right)$ as graded functions on $E[1]$ (of degree n), which can further be pulled back to the minimal symplectic realization $\pi: \mathcal{E} \rightarrow E[1]$. Thus, we can identify $\Gamma\left(\Lambda^{n}(\operatorname{ker} \rho)\right)$ with a subalgebra of $\mathcal{C}^{\infty}(\mathcal{E})$. Stiénon-Xu proved [SX08] the following Proposition.

Proposition 3.3. The Q-structure $Q=\{H,$.$\} (see Definition 2.5) maps$ $\Gamma\left(\Lambda^{\bullet}(\operatorname{ker} \rho)\right)$ to itself. Moreover, if $\alpha \in \Gamma\left(\Lambda^{\bullet}(\operatorname{ker} \rho)\right)$, then $Q(\alpha)=d(\alpha)$.

In other words, $Q$ restricted to $\Gamma\left(\Lambda^{\bullet}(\right.$ ker $\left.\rho)\right)$ coincides with the differential $d$ given by formula (3.1) (note that Proposition 3.3 also implies that $d \circ d=$ $0)$. Since $d$ squares to 0 , Stiénon-Xu defined :

Definition 3.4 (Naive cohomology). Let $(E,[.,],. \rho,\langle.,\rangle$.$) be a Courant alge-$ broid. The naive cohomology of $E$ is the cohomology of the sections of $\Lambda^{\bullet}$ ker $\rho$ equipped with the differential $d$ given by the Cartan-formula (3.1).

We denote $H_{\text {naive }}^{\bullet}(E)=H^{\bullet}\left(\Gamma\left(\Lambda^{\bullet} \operatorname{ker} \rho\right), d\right)$ the naive cohomology groups of $E$. By Proposition 3.3, there is a canonical morphism $\phi: H_{\text {naive }}^{\bullet}(E) \rightarrow$ $H_{\text {std }}^{\bullet}(E)$ from the naive cohomology to the (standard) cohomology of Courant algebroids, see [SX08]. We will prove that this morphism is an isomorphism in the transitive case, see Corollary 4.10.

\section{Geometric spectral sequence for cohomology of Courant algebroids with split base}

In this section we define a spectral sequence converging to the cohomology of a Courant algebroid. Then, in the case of Courant algebroids with split base, we compute the spectral sequence in terms of geometrical data. For details about spectral sequences refer to [CEal, McC85].

\footnotetext{
${ }^{4}$ In this case $\Gamma(\operatorname{ker} \rho)$ means smooth sections into $E$ that are pointwise in the kernel of $\rho$. We define similarly sections of $\Lambda^{\bullet} \operatorname{ker} \rho$ where $\rho$ has been extended as an odd $\mathcal{C}^{\infty}(M)$ linear derivation $\Lambda^{\bullet} E \rightarrow \Lambda^{\bullet} E \otimes T M$.
} 
4.1. The naive ideal spectral sequence. The algebra $A^{\bullet}:=C^{\infty}(\mathcal{E})$ of graded functions on $\mathcal{E}$ is endowed with a natural filtration induced by the ideal generated by the kernel of the anchor map $\rho: E \rightarrow T M$. More precisely, let $I$ be the ideal

$$
I:=\Gamma\left(\Lambda^{\geq 1} \operatorname{ker} \rho\right) \cdot C^{\infty}(\mathcal{E}),
$$

i.e., the ideal of functions containing at least one coordinate of ker $\rho$, where we identify sections of $E$ to odd functions on $E$ by the pseudo-metric (as in Section 3). Since $\operatorname{ker} \rho$ gives rise to the naive cohomology, we call $I$ the naive ideal of $\mathcal{E}$.

Lemma 4.1. I is a differential ideal of the differential graded algebra $\left(A^{\bullet}, Q\right)$

Proof. According to Proposition 3.3 and Lemma 3.1, we have $Q\left(\Gamma\left(\Lambda^{n} \operatorname{ker} \rho\right)\right)$ $\subset \Gamma\left(\Lambda^{n+1} \operatorname{ker} \rho\right)$. The result follows since $Q$ is a derivation.

Since $I$ is a differential ideal, we have a decreasing bounded (since $E$ is finite dimensional) filtration of differential graded algebras $A^{\bullet}=F^{0} A^{\bullet} \supset$ $F^{1} A^{\bullet} \supset F^{2} A^{\bullet} \ldots$, where $F^{p} A^{q}:=I^{p} \cap A^{q}$. Therefore :

Proposition 4.2. There is a spectral sequence of algebras

$$
E_{0}^{p, q}:=F^{p} A^{p+q} / F^{p+1} A^{p+q} \Longrightarrow H_{\text {std }}^{p+q}(E)
$$

converging to the cohomology of the Courant algebroid E.

We call this spectral sequence, the naive ideal spectral sequence.

Proof. The spectral sequence is the one induced by the filtration $F^{\bullet} A^{\bullet}$ of the complex $\left(A^{\bullet}, Q\right)$. It is convergent because the filtration is bounded.

Remark 4.3. In order for the naive ideal spectral sequence to be useful, one needs to be able to calculate the higher sheets $E_{k}^{p, q}$ of the spectral sequence in terms of (smooth) geometry of $E$. Such calculations involve the image of the anchor map. Thus it seems reasonable to restrict to the class of regular Courant algebroids.

4.2. Courant algebroids with split base. In this section we compute explicitly the naive ideal spectral sequence of Proposition 4.2 for what we call Courant algebroid with split base and then prove the conjecture of Stiénon-Xu as a special case.

By the bracket preserving-property of the anchor-map $\rho, D:=\operatorname{im} \rho$ is an integrable (possibly singular) distribution.

Definition 4.4 (Split base). A Courant algebroid $(E \rightarrow M,\langle.,\rangle,.[.,],. \rho)$ is said to have split base iff $M \cong L \times N$ and the image of the anchor map is $D:=\operatorname{im} \rho \cong T L \times N \subset T M$. 
In particular, a Courant algebroid with split base is a regular Courant algebroid. Furthermore, the integral leaves of the distribution $D$ are smoothly parametrized by the points of $N$; thus the quotient $M / D$ by the integral leaves of the foliation is isomorphic to $N$.

Remark 4.5. The local coordinates $\xi^{a}$ for $E$ introduced in Section 2 can be splitted accordingly to the isomorphism $D \cong T L \times N$. This splitting is useful in order to do local computations. More precisely, over a chartneighborhood $U$ of $M, D:=\operatorname{im} \rho$ can be spanned by coordinate vector fields $\frac{\partial}{\partial x^{I}}$. Let $\xi^{I}:=\rho^{*} d x^{I}(\in \operatorname{ker} \rho)$ be vectors that span the image of $\rho^{*}$, and let $\xi_{I}$ be preimages of $\partial_{I}$, dual to the $\xi^{I}$. Then choose coordinates $\xi^{A} \in \operatorname{ker} \rho$ normal (with respect to the pseudo-metric) and orthogonal to both the $\xi^{I}$ 's and the $\xi_{I}$ 's. Therefore we have split the coordinates $\xi^{a}$ 's in the three subsets consisting of the $\xi^{I}$ 's, the $\xi_{I}$ 's and the $\xi^{A}$ 's. Furthermore, this splitting also induces a splitting of the degree 2 coordinates $p_{i}$ 's (the conjugates of the coordinates on $M$ ) into the $p_{I}$ 's, which are the symplectic duals of the $x^{I}$, and the $p_{I}$ 's (their complements for which the Poisson bracket with the $x^{I}$ 's vanish). Since $D \cong T L \times N$, the coordinates $x^{I}$ and $x^{I}$, can be chosen to be coordinates of $L$ and $N$, respectively.

The Hamiltonian in these coordinates reads as $H=p_{I} \xi^{I}+\frac{1}{6} C_{a b c}(x) \xi^{a} \xi^{b} \xi^{c}$. Note that in order to compute structure functions like $C_{a b c}$ you need $\xi_{A}$ which is the dual frame of $\xi^{A}$ or due to the pseudo orthonormality $\xi_{A}= \pm \xi^{A}$.

We denote $\mathcal{X}^{q}(N)$ the space of (degree $q$ ) symmetric multivector fields $\Gamma_{N}\left(S^{q / 2}(T N)\right)$ with the convention that $S^{q / 2}(T N)$ is $\{0\}$ for odd $q$ 's, i.e., $\mathcal{X}^{\bullet}(N)$ is concentrated in even degrees. With these notations, the sheet $E_{1}^{\bullet, \bullet}$ of the spectral sequence of Proposition 4.2 is given by:

Lemma 4.6. For a Courant algebroid with split base $D \cong T L \times N$, one has

$$
E_{1}^{p, q} \cong \Gamma_{M} \Lambda^{p}(\operatorname{ker} \rho) \otimes \mathcal{X}^{q}(N) .
$$

Proof. The differential $d_{0}: E_{0}^{p, q} \rightarrow E_{0}^{p, q+1}$ is the differential induced on the associated graded $\bigoplus F^{p} A^{p+q} / F^{p+1} A^{p+q}$ of the naive filtration $F^{\bullet} A^{\bullet}$. Hence, it is obtained from $Q$ by neglecting all terms which contain at least one term in $I$. Using the local coordinates of Remark 4.5, we find $d_{0}=p_{I} \frac{\partial}{\partial \xi_{I}}$. Globally, the algebra $E_{0}^{0, \bullet}$ is isomorphic to $\mathcal{C}^{\infty}(B)$ for the graded manifold $B:=\mathcal{E} / I$ which, on a local chart $U \subset M$ is isomorphic to $B_{\mid U} \cong T^{*}[2] U \otimes_{U} D[1] \mid U$. Globally, $B$ fits into the short exact sequence of graded fiber bundles over $M$ :

$$
0 \rightarrow T^{*}[2] M \rightarrow B \rightarrow D[1] \rightarrow 0,
$$

where the latter map $B \rightarrow D[1]$ is the map induced by the anchor map $\rho: E \rightarrow D=\operatorname{im} \rho$ on the quotient of $\mathcal{E}$ by $I$. The differential $d_{0}$ on $E_{0}^{0, \bullet} \cong$ $\mathcal{C}^{\infty}(B)$ is canonically identified with the odd vector field $\tilde{\rho}_{0}$ induced by $Q$ on the quotient $B=\mathcal{E} / I$. 
There is a similar interpretation of $E_{0}^{1, \bullet}$. Precisely, $E_{0}^{1, \bullet}$ is isomorphic to $\Gamma_{B}\left(B_{1}\right)$ for a (graded) vector bundle $B^{1} \rightarrow B$ which, locally, is the fiber product $B_{\mid U}^{1} \cong \operatorname{ker} \rho[1] \times_{U} B$. In particular, $B^{1}$ fits into the short exact sequence of graded fiber bundles over $M$ :

$$
0 \rightarrow \operatorname{ker} \rho[1] \rightarrow B^{1} \rightarrow B \rightarrow 0 .
$$

The isomorphism $E_{0}^{1, \bullet} \cong \Gamma_{B}\left(B_{1}\right)$ identifies the differential $d_{0}: E_{0}^{1, \bullet} \rightarrow$ $E_{0}^{1, \bullet+1}$ with the odd vector field $\tilde{\rho}: \Gamma_{B}\left(B^{1}\right) \rightarrow \Gamma_{B}\left(B^{1}\right)$ defined as the covariant derivative $\tilde{\rho}=\nabla_{\tilde{\rho}_{0}}$ along $\tilde{\rho}_{0}$ where $\nabla$ is a local connection on $B_{1}$ vanishing on a local frame $\xi^{a}$ of $B^{1}$. This is well defined, because the transition functions between such frames come from functions on $M$ and $\tilde{\rho_{0}}$ projected to $M$ vanishes (we extend to arbitrary sections of $B^{1}$ via Leibniz rule).

This identification of $E_{0}^{1, \bullet}$ extends to the other lines $E_{0}^{p \geq 2, \bullet}$ of the spectral sequence easily. Namely, there is an isomorphism $E_{0}^{p, \bullet} \cong \Gamma_{B}\left(S^{p} B^{1}\right)$, where $S^{p}$ stands for the graded symmetric (hence it is skew-symmetric since the fibers ker $\rho[1]$ are of odd degree) product over $B$. We extend $\tilde{\rho}$ to $\Gamma_{B}\left(S^{p} B^{1}\right)$ by the Leibniz rule. Since, by Lemma $4.2, d_{0}$ is a derivation, we also have the identification of $d_{0}: E_{0}^{p \geq 2, \bullet} \rightarrow E_{0}^{p \geq 2, \bullet+1}$ and $\tilde{\rho}$.

According to Lemma $4.2, d_{0}$ is a derivation, thus it is sufficient to compute the cohomology of the complex $\left(E_{0}^{0, \bullet}, d_{0}\right)$. Sequence (4.1) yields a morphism of sheaves $\eta: \mathcal{C}^{\infty}(B) \rightarrow \Gamma_{M}\left(S^{\bullet}(T M)[2]\right) \rightarrow \Gamma_{M}\left(S^{\bullet}(T M / D)[2]\right)$. On a local chart, the complex $\left(E_{0}^{0, \bullet}, d_{0}\right)$ is isomorphic to the Koszul complex of $\mathcal{B}^{\bullet}:=$ $\Gamma_{M}\left(S^{\bullet}(T M)[2] \otimes \Lambda^{\bullet}(E / \operatorname{ker} \rho)\right)$ with respect to the differential $\tilde{\rho}=p_{I} \frac{\partial}{\partial \xi_{I}}$ induced by the regular family given by the $p_{I}$ 's. Since the image of $\tilde{\rho}$ spans $D \cdot \mathcal{B}^{\bullet}$, the morphism of sheaves $\eta: \mathcal{C}^{\infty}(B) \rightarrow \Gamma_{M}\left(S^{\bullet}(T M / D)[2]\right)$ is locally a quasi-isomorphism. Thus we have

$$
\begin{aligned}
H^{q}\left(E_{0}^{0, \bullet}, d_{0}\right) & \cong \Gamma_{M}\left(S^{\bullet}(T M / D)[2]\right) \\
& \cong \Gamma_{N}\left(S^{\bullet} T(N)[2]\right) \otimes_{\mathcal{C}^{\infty}(N)} \mathcal{C}^{\infty}(L \times N) \\
& \cong \mathcal{X}^{q}(N) \otimes_{\mathcal{C}^{\infty}(N)} \mathcal{C}^{\infty}(M),
\end{aligned}
$$

where the second line follows from the fact that $E$ has split base $D \cong T L \times N$. The computation of the line $E_{1}^{1, \bullet}$ is similar since $\tilde{\rho}$ is a horizontal lift of $\tilde{\rho}_{0}$. In particular, $\tilde{\rho}$ does not act on the fibers ker $\rho[1]$ of $B^{1} \rightarrow B$, but only on the base $B$. Hence

$$
H^{q}\left(E_{0}^{1, \bullet}, d_{0}\right) \cong \mathcal{X}^{q}(N) \otimes \Gamma_{M}(\operatorname{ker} \rho) .
$$

Since the lines, $E_{0}^{p, 0}$ are generated by (products of elements of) $E_{0}^{1,0}$ and $d_{0}$ is a derivation, the result follows.

Remark 4.7. Lemma 4.6 is the main reason to restrict to Courant algebroids with split base. In general, one can consider the quotient $M / D$ of 
$M$ by the integral leaves of the integrable distribution $D:=\operatorname{im} \rho$. Let $\mathcal{C}^{\infty}(M)^{D}$ be the space of smooth functions on $M$ constant along the leaves and $\mathcal{X}_{\text {flat }}(M / D)$ the space of derivations of $\mathcal{C}^{\infty}(M)^{D}$. To describe the spectral sequence using smooth geometry, we would like a formula of the form

$$
E_{1}^{p, q} \cong \Gamma_{M}\left(\Lambda^{p} \operatorname{ker} \rho\right) \otimes S^{q / 2}\left(\mathcal{X}_{\text {flat }}(M / D)\right) .
$$

A quick analysis of the proof of Lemma 4.6 shows that this formula will hold if and only if we have the relation

$$
\Gamma_{M}(T M / D) \cong \Gamma_{M / D}\left(T_{\text {flat }}(M / D)\right) \otimes_{\mathcal{C}^{\infty}(M)^{D}} \mathcal{C}^{\infty}(M)
$$

Courant algebroids with split base are a large class for which relation (4.3) holds. However relation (4.3) does not hold for every regular Courant algebroid. For instance, take the Lie algebroid $D$ underlying the irrational torus. That is $M=\mathbb{T}^{2}$ is foliated by the action of a non-compact one parameter subgroup of $\mathbb{T}^{2}$ and $D$ is the subbundle of $T M$ associated to the foliation. The leaves are dense. Let $E=D \oplus D^{*}$ be a generalized exact Courant algebroid (as in Example 5.5). Note that $D$ is regular of rank 1, thus $\Gamma_{M}(T M / D)$ is non-zero but $\mathcal{C}^{\infty}(M)^{D} \cong \mathbb{R}$, thus $\mathcal{X}_{\text {flat }}(M / D)=0$. Thus formula (4.3) does not hold for $E$.

Recall from Section 3 that the naive cohomology $H_{\text {naive }}^{\bullet}(E)$ is the cohomology of the complex $\left(\Gamma_{M} \Lambda^{\bullet}(\operatorname{ker} \rho), d\right)$. The second sheet of the spectral sequence is computed by $H_{\text {naive }}^{\bullet}(E)$ :

Proposition 4.8. Let $E$ be a Courant algebroid with split base $D \cong T L \times N$. Then, one has an isomorphism of graded algebras

$$
E_{2}^{p, q} \cong H_{\text {naive }}^{p}(E) \otimes \mathcal{X}^{q}(N) .
$$

Proof. It is a standard fact of spectral sequences [CEal, McC85] that the differential

$$
d_{1}: E_{1}^{p, q} \cong H^{q}\left(F^{p} A / F^{p+1} A, d_{0}\right) \rightarrow E_{1}^{p+1, q} \cong H^{q}\left(F^{p+1} A / F^{p+2} A, d_{0}\right)
$$

is the connecting homomorphism in the cohomology long exact sequence induced by the short exact sequence of complexes $0 \rightarrow F^{p+1} A / F^{p+2} A \rightarrow$ $F^{p} A / F^{p+2} A \rightarrow F^{p} A / F^{p+1} A \rightarrow 0$. On a local chart, we obtain that $d_{1}$ is given by the following formula

$$
\begin{aligned}
d_{1}= & \xi^{I} \frac{\partial}{\partial x^{I}}+C_{I A}^{K} \xi^{I} \xi^{A} \frac{\partial}{\partial \xi^{K}}+\frac{1}{2} C_{A B}^{K} \xi^{A} \xi^{B} \frac{\partial}{\partial \xi^{K}}+\frac{1}{2} C_{I J}^{A} \xi^{I} \xi^{J} \frac{\partial}{\partial \xi^{A}} \\
& +C_{A I}^{B} \xi^{A} \xi^{I} \frac{\partial}{\partial \xi^{B}}+\frac{1}{2} C_{A B}^{C} \xi^{A} \xi^{B} \frac{\partial}{\partial \xi^{C}}
\end{aligned}
$$

where we use the local coordinates introduced in Remark 4.5. Now it follows from the isomorphism $E_{1}^{p, q} \cong \Gamma_{M} \Lambda^{p}(\operatorname{ker} \rho) \otimes \mathcal{X}^{q}(N)$ given by Lemma 4.6 
and the above formula for $d_{1}$ that

$$
d_{1}=d \otimes 1: \Gamma_{M} \Lambda^{p}(\operatorname{ker} \rho) \otimes \mathcal{X}^{q}(N) \rightarrow \Gamma_{M} \Lambda^{p+1}(\operatorname{ker} \rho) \otimes \mathcal{X}^{q}(N),
$$

where $d$ is the naive differential. The result follows.

The third sheet of the spectral sequence is trivially deduced from Proposition 4.8 .

Corollary 4.9. Let $E$ be a Courant algebroid with split base. There is a canonical isomorphism of bigraded algebras $E_{3}^{\bullet, \bullet} \cong E_{2}^{\bullet, \bullet}$.

Proof. Since $\mathcal{X}^{q}(N)$ is concentrated in even degrees for $q$, so is $E_{2}^{p, q}$ by Proposition 4.8. Therefore, the differential $d_{2}: E_{2}^{p, q} \rightarrow E_{2}^{p+2, q-1}$ is necessarily 0 . Hence $E_{3}^{\bullet, \bullet} \cong E_{2}^{\bullet, \bullet}$.

We now prove the conjecture of Stiénon-Xu. There is a canonical morphism $\phi: H_{\text {naive }}^{\bullet}(E) \rightarrow H_{\text {std }}^{\bullet}(E)$, see [SX08] and Section 3 .

Corollary 4.10. Let $E$ be a transitive Courant algebroid. Then the canonical map $\phi$ is an isomorphism $\phi: H_{\text {naive }}^{\bullet}(E) \cong H_{\text {std }}^{\bullet}(E)$, i.e., the Courant algebroid cohomology coincides with the naive cohomology.

Proof. A transitive Courant algebroid satisfies $D:=\operatorname{im} \rho=T M$. Therefore, $E$ is trivially with split base and $M / D=\mathrm{pt}=N$. Hence $\mathcal{X}^{q}(N)$ is non-zero only for $q=0$, where it is $\mathbb{R}$. Therefore, by Proposition $4.8, E_{2}^{p, q}=0$ if $q \neq 0$. It follows that all the higher differentials $d_{n \geq 3}$ are null. Thus the cohomology of the Courant algebroid is isomorphic to $E_{2}^{\bullet, 0} \cong H_{\text {naive }}^{\bullet}(E) \otimes \mathbb{R}$.

Furthermore, by definition of the naive ideal $I$, the map $\phi$ preserves the filtration by $I$. Thus $\phi$ passes to the spectral sequence and coincides with the morphism of complexes $\phi_{1}:\left(\Gamma\left(\Lambda^{n} \operatorname{ker} \rho\right), d\right) \cong\left(E_{1}^{n, 0}, d_{1}\right) \hookrightarrow$ $\left(\oplus_{p+q=n} E_{1}^{p, q}, d_{1}\right)$ on the first sheet of the spectral sequence. By the first paragraph of this proof, $\phi_{1}$ is a quasi-isomorphism. Hence $\phi$ is indeed an isomorphism.

Remark 4.11. When $E$ is an exact Courant algebroid, Corollary 4.10 was obtained by Roytenberg [Roy01]. Indeed, it was one of the examples motivating the conjecture of Stiénon-Xu.

For general Courant algebroids $E$ with split base, the spectral sequence does not collapse on the sheet $E_{2}^{\bullet \bullet \bullet}$ but is controlled by a map from the vector fields on $N$ to the naive cohomology of $E$ which we now describe.

It is a general fact from the spectral sequence theory that there exists a differential $d_{3}: E_{3}^{p, q} \rightarrow E_{3}^{p+3, q-2}$. In particular, $d_{3}$ induces an $E_{3}^{0,0}=\mathcal{C}^{\infty}(N)$ linear map

$$
T_{3}: \mathcal{X}(N) \rightarrow H_{\text {naive }}^{3}(E)
$$


given as the composition

$$
T_{3}: \mathcal{X}(N) \cong \mathcal{X}^{2}(N) \cong E_{3}^{0,2} \stackrel{d_{3}}{\longrightarrow} E_{3}^{3,0} \cong H_{\text {naive }}^{3}(E) .
$$

We call the map $T_{3}$ the transgression homomorphism of the Courant algebroid $E$. Let $\mathcal{X}^{\text {kil }}(N)$ be the kernel of $T_{3}$ above (we like to think of elements of $\mathcal{X}^{\mathrm{kil}}(N)$ as Killing vector fields preserving the structure function $\left.H\right)$. Note that $\mathcal{X}^{\text {kil }}(N)$ may be singular, i.e., its rank could vary. We denote $\mathcal{X}^{\text {kil,q }}$ the space of "symmetric Killing multivector fields" $S_{\mathcal{C}^{\infty}(N)}^{q / 2}\left(\mathcal{X}^{\text {kil }}(N)\right)$ with the convention that $\mathcal{X}^{\mathrm{kil}, q}=\{0\}$ for odd $q$.

Theorem 4.12. The cohomology of a Courant algebroid E with split base is given by

$$
H_{\mathrm{std}}^{n}(E) \cong \bigoplus_{p+q=n} H_{\text {naive }}^{p}(E) /\left(T_{3}\right) \otimes \mathcal{X}^{\mathrm{kil}, q},
$$

where $\left(T_{3}\right)$ is the ideal in $H_{\text {naive }}^{\bullet}(E)$ which is generated by the image $T_{3}(\mathcal{X}(N))$ of $T_{3}$.

Proof. According to Proposition 4.8 and Corollary 4.9, the third sheet of the spectral sequence is given by $E_{3}^{p, q} \cong H_{\text {naive }}^{p}(E) \otimes \mathcal{X}^{q}(N)$. Note that $\mathcal{X}^{q}(N)=\Gamma_{N}\left(S^{q / 2}(T N)\right)$ is generated as an algebra by its degree 2 elements. Since the differential $d_{3}: E_{3}^{p, q} \rightarrow E_{3}^{p+3, q-2}$ is a derivation, it is necessarily the unique derivation extending its restriction $T_{3}=d_{3}: \mathcal{X}(N) \rightarrow H_{\text {naive }}^{3}(E)$. Since $\mathcal{X}^{\bullet}(N)=\Gamma_{N}\left(S^{q / 2}(T N)\right)$ is a free graded commutative algebra, the cohomology $E_{4}^{\bullet, \bullet}=H^{\bullet}\left(E_{3}^{\bullet, \bullet}, d_{3}\right)$ is given by

$$
\begin{aligned}
E_{4}^{\bullet, q} & \cong H_{\text {naive }}^{\bullet}(E) /\left(\operatorname{im}\left(T_{3}\right)\right) \otimes S^{q / 2}\left(\operatorname{ker} T_{3}\right) \\
& \cong H_{\text {naive }}^{\bullet}(E) /\left(T_{3}\right) \otimes \mathcal{X}^{\mathrm{kil}, q} .
\end{aligned}
$$

Now, it is sufficient to prove that all higher differentials $d_{r \geq 4}$ vanish. Since $d_{4}: E_{4}^{p, q} \rightarrow E_{4}^{p+4, q-3}$ is a derivation, it is completely determined by its restriction to the generators of $E_{4}^{\bullet \bullet \bullet}$ which lie in $E_{4}^{\bullet, 0}$ and $E_{4}^{0,2}$. Thus, for obvious degree reasons, $d_{4}=0$ and similarly for all $d_{r>4}$. Therefore, the spectral sequence collapses at the fourth sheet: $E_{\infty}^{p, q} \cong E_{4}^{p, q}$.

Remark 4.13. Theorem 4.12 gives an isomorphism of vector spaces, not of algebras in general. However, by standard results on spectral sequences of algebras, isomorphism (4.5) is an isomorphism of graded algebras if the right-hand side of (4.5) is free as a graded commutative algebra.

Remark 4.14. Theorem 4.12 implies that all the cohomological information of a Courant algebroid with split base is encoded in the transgression homomorphism $T_{3}$ together with the naive cohomology (and image of the anchor map). We like to think of $T_{3}$ as a family of closed 3-sections of ker $\rho$ obtained by transgression from $\mathcal{X}(N)$. This idea is made more explicit in 
the case of generalized exact Courant algebroids in Section 5. In that case, the transgression homomorphism is closely related to a generalization of the characteristic class of the Courant algebroids as defined by Ševera [ک̌ev99], that is the cohomology class of the structure 3-form (see Propositions 5.2 and 5.6) parameterizing such Courant algebroids.

\section{Generalized exact Courant algebroids with split base}

In this section, we consider a generalization of exact Courant algebroids. These Courant algebroids are parametrized by the cohomology class of closed 3 -forms from which an explicit formula for the transgression homomorphism $T_{3}$ can be given.

An exact Courant algebroid $E \rightarrow M$ is a Courant algebroid such that the following sequence

$$
0 \rightarrow T^{*} M \stackrel{\rho^{*}}{\rightarrow} E \stackrel{\rho}{\rightarrow} T M \rightarrow 0
$$

is exact.

Assume that $D:=\operatorname{im} \rho \subset T M$ is a subbundle, i.e, $E$ is regular. Then the anchor maps surjectively $E \stackrel{\rho}{\rightarrow} D$ and its dual $\rho^{T}: T^{*} M \rightarrow E^{*} \cong E$ factors

through an injective map $D^{*} \stackrel{\rho^{*}}{\rightarrow} E$ (again $E$ and $E^{*}$ are identified by the pseudo-metric).

Definition 5.1. A regular Courant algebroid $(E, \rho, \ldots)$ is generalized exact if the following sequence of bundle morphisms over $M$

$$
0 \rightarrow D^{*} \stackrel{\rho^{*}}{\rightarrow} E \stackrel{\rho}{\rightarrow} D \rightarrow 0
$$

is exact.

By the above discussion, the only condition to check for sequence (5.1) to be exact is exactness in $E$.

There is a simple geometric classification of exact Courant algebroids due to Ševera [Šev99] which extends easily to generalized exact Courant algebroids as follows. Note that $D$ is Lie subalgebroid of $T M$. Given a Lie algebroid $D$, we write $\left(\Omega^{\bullet}(D), d_{D}\right)$ the complex of $D$-forms, $H_{\text {Lie }}^{\bullet}(D)$ its cohomology and $Z^{\bullet}(D)=\operatorname{ker}\left(d_{D}\right)$ the closed $D$-forms [Mac87, SW99].

Let $(E \rightarrow M, \rho,[.,],.\langle.,\rangle$.$) be a generalized exact Courant algebroid and$ assume given a splitting of the exact sequence (5.1) as a pseudo-Euclidean vector bundle, that is, an isotropic (with respect to the pseudo-metric) section $\sigma: D \rightarrow E$ of $\rho$. Thus the section $\sigma$ identifies $E$ with $D^{*} \oplus D$ endowed with its standard pseudo-metric: $\langle\alpha \oplus X, \beta \oplus Y\rangle=\alpha(Y)+\beta(X)$. Since $\rho$ preserves the bracket, for any $X, Y \in \Gamma(D)$, one has

$$
[\sigma(X), \sigma(Y)]=\sigma\left([X, Y]_{T M}\right) \oplus \tilde{C}_{\sigma}(X, Y),
$$

where $\tilde{C}_{\sigma}(X, Y) \in \rho^{*}\left(D^{*}\right)$. Let $C_{\sigma}$ be the dual of $\tilde{C}_{\sigma}$, that is, for $X, Y, Z \in$ $\Gamma(D)$, we define $C_{\sigma}(X, Y, Z)=\left\langle\tilde{C}_{\sigma}(X, Y), Z\right\rangle$. It follows from axioms (2.3) 
and (2.4) of a Courant algebroid (see Definition 2.1) that $C_{\sigma}$ is skewsymmetric. Moreover, by axioms (2.2) and (2.4), $C_{\sigma}$ is $\mathcal{C}^{\infty}(M)$-linear. Thus $C_{\sigma}$ is indeed a 3 -form on the Lie algebroid $D$, i.e., $C_{\sigma} \in \Omega^{3}(D)$. Furthermore, the (specialized) Jacobi identity, i.e., axiom (2.1) implies that $C_{\sigma}$ is closed, that is, $d_{D}\left(C_{\sigma}\right)=0$.

Proposition 5.2 (The Ševera characteristic class). Let $E$ be a generalized exact Courant algebroid.

(1) There is a splitting of the exact sequence (5.1) as a pseudo-Euclidean bundle; in particular, there is an isotropic section $\sigma: D \rightarrow E$ of $\rho$.

(2) If $\sigma^{\prime}: D \rightarrow E$ is another isotropic section, then $C_{\sigma}-C_{\sigma^{\prime}}$ is an exact 3-form, that is, $C_{\sigma}-C_{\sigma^{\prime}} \in \operatorname{im} d_{D}$.

Proof. The proof is the same as the one for exact Courant algebroids, for instance, see Ševera Letter [Šev99] or [Roy99, Section 3.8].

In particular the cohomology class $\left[C_{\sigma}\right] \in H_{\text {Lie }}^{3}(D)$ is independent of $\sigma$. We will simply denote it $[C]$ henceforth. We call the class $[C] \in H_{\text {Lie }}^{3}(D)$ the Ševera class of $(E \rightarrow M, \rho,[.,],.\langle.,\rangle$.$) .$

Given a closed 3-form $C \in \Omega^{3}(D)$, one can define a bracket on the pseudoEuclidean vector bundle $D^{*} \oplus D$ given, for $X, Y \in \Gamma(D), \alpha, \beta \in \Gamma\left(D^{*}\right)$, by the formula

$$
[\alpha \oplus X, \beta \oplus Y]=\mathcal{L}_{X} \beta-\imath_{Y} d_{D}(\alpha)+C(X, Y, .) \oplus[X, Y]_{T M} .
$$

It is straightforward to check that this bracket makes the pseudo-Euclidean bundle $D^{*} \oplus D$ a Courant algebroid, where the anchor map is the projection $D^{*} \oplus D \rightarrow D$ [Roy99, Section 3.8]. Clearly its Ševera class is $C$. Moreover two cohomologous closed 3-forms $C, C^{\prime} \in \Omega^{3}(D)$ yield isomorphic Courant algebroids [Roy99, Section 3.8]. Therefore

Proposition 5.3 (Analog of the Ševera classification). Let D be a Lie subalgebroid of a smooth manifold $M$. The isomorphism classes of generalized exact Courant algebroids with fixed image $\operatorname{im} \rho=D$ are in one-to-one correspondence with $H_{\text {Lie }}^{3}(D)$, the third cohomology group of the Lie algebroid $D$.

The correspondence assigns to a Courant algebroid E its Ševera characteristic class $[C]$.

Remark 5.4. Generalized exact Courant algebroids are easy to describe in terms of the derived bracket construction. Indeed, since we can choose an isomorphism $E \cong D \oplus D^{*}$, the minimal symplectic realization of $E$ is isomorphic to $T^{*}[2] D[1]$. From the explicit formula (5.2) for the Courant bracket, we found that the generating cubic Hamiltonian $H$ (encoding the Courant algebroid structure) is given, in our adapted coordinates, by

$$
H=p_{I} \xi^{I}+\frac{1}{6} C_{I J K} \xi^{I} \xi^{J} \xi^{K}
$$


where $C_{I J K}$ are the components of the Ševera 3 -form $C$ induced by the splitting $\cong D \oplus D^{*}$.

Now assume that $E$ is a generalized exact Courant algebroid with split base $D \cong T L \times N$. Then, there is an isomorphism $\Omega^{\bullet}(D) \cong \Omega^{\bullet}(L) \otimes_{\mathbb{R}} \mathcal{C}^{\infty}(N)$ and the de Rham differential $d_{D}$ of the Lie algebroid $D$ is identified with $d_{L} \otimes 1$, the de Rham differential of the smooth manifold $L$, see Remark 3.2. In particular, $Z^{3}(D) \cong Z^{3}(L) \otimes_{\mathbb{R}} \mathcal{C}^{\infty}(N)$. Furthermore, since ker $\rho \cong D^{*} \cong$ $T^{*} L \times N$, the naive complex $\left(\Gamma_{M}\left(\Lambda^{\bullet} \operatorname{ker} \rho\right), d\right)$ is isomorphic to the complex $\left(\Omega^{\bullet}(L) \otimes \mathcal{C}^{\infty}(N), d_{L} \otimes 1\right)$ of de Rham forms on $L$ tensored by smooth functions on $N$. Therefore $H_{\text {naive }}^{\bullet}(E) \cong H_{\mathrm{DR}}^{\bullet}(L) \otimes \mathcal{C}^{\infty}(N)$, where $H_{D R}^{\bullet}(L)$ is the de Rham cohomology of the manifold $L$.

Example 5.5. Let $L$ and $N$ be two smooth manifolds and define $M:=$ $L \times N, D:=T L \times N$ which is a subbundle of $T M$. Pick $\omega \in Z^{3}(L)$ any closed 3-form on $L$ and $f \in \mathcal{C}^{\infty}(N)$ be any function on $N$. Then $C:=\omega \otimes f$ is a closed 3-form on $D$. The 3 -form $C$ induces a generalized exact Courant algebroid with split base structure on $E:=D \oplus D^{*}$ where the Courant bracket is given by formula (5.2), the pseudo-metric is the standard pairing between $D$ and $D^{*}$ and the anchor map the projection $E \rightarrow D$ on the first summand. By Proposition 5.3, any generalized exact Courant algebroid with split base is isomorphic to such a Courant algebroid.

For any vector field $X \in \mathcal{X}(N)$ on $N$, there is the map

$$
\Omega_{\text {naive }}^{\bullet}(E) \cong \Omega^{\bullet}(L) \otimes \mathcal{C}^{\infty}(N) \stackrel{1 \otimes X}{\longrightarrow} \Omega^{\bullet}(L) \otimes \mathcal{C}^{\infty}(N) \cong \Omega_{\text {naive }}^{\bullet}(E)
$$

defined, for $\omega \in \Omega^{\bullet}(L)$ and $f \in \mathcal{C}^{\infty}(N)$ by $(1 \otimes X)(\omega \otimes f)=\omega \otimes X[f]$. Applying this map to the Ševera 3 -form $C \in Z^{3}(L) \otimes \mathcal{C}^{\infty}(N) \cong Z^{3}(D)$ yields the map

$$
\mathcal{X}(N) \ni X \mapsto[(1 \otimes X)(C)] \in H_{\text {naive }}^{3}(E)
$$

which is well defined and depends only on the Ševera class of $E$ (and not on the particular choice of a 3-form representing it) by Proposition 5.2.

Proposition 5.6. Let $E$ be a generalized exact Courant algebroid with Ševera class $[C] \in H_{\text {Lie }}^{3}(D) \cong H_{\mathrm{DR}}^{3}(L) \otimes \mathcal{C}^{\infty}(N)$. The transgression homomorphism $T_{3}: \mathcal{X}(N) \rightarrow H_{\text {naive }}^{3}(E)$ is the map given, for any vector field $X \in \mathcal{X}(N)$, by

$$
T_{3}(X)=[(1 \otimes X)(C)] \in H_{\text {naive }}^{3}(E) .
$$

Proof. Fix a Ševera 3 -form $C$ representing the Ševera characteristic class. The transgression homomorphism is induced by the differential $d_{3}: E_{3}^{\bullet, \bullet} \rightarrow$ $E_{3}^{\bullet+3, \bullet-2}$. Corollary 4.9 and Proposition 4.8 together with above remarks about generalized exact Courant algebroids give: $E_{3}^{p, q} \cong \Gamma\left(\Lambda^{p} \mathrm{im} \rho^{*}\right) \otimes$ 
$\mathcal{X}^{q}(N)$. Therefore our adapted coordinates 4.5 still apply and give on a local chart the map $d_{3}$ as

$$
d_{3}=\frac{1}{6} C_{I J K, L^{\prime}} \xi^{I} \xi^{J} \xi^{K} \frac{\partial}{\partial p_{L^{\prime}}}
$$

By formula (5.3), the functions $\frac{1}{6} C_{I J K} \xi^{I} \xi^{J} \xi^{K}$ are given by the components of the Ševera 3 -form $C$ (identified with a function on $E[1]$ via $\langle.,$.$\rangle and$ pulled back to $\mathcal{E})$. Now the result follows since $T_{3}$ is the restriction of $d_{3}$ to $E_{3}^{0,2} \cong \mathcal{X}(N)$.

We denote $\operatorname{Ann}(C)$ the kernel of $T_{3}$, that is the vector fields $X$ on $N$ such that $[(1 \otimes X)(C)]=0 \in H_{\text {naive }}^{3}(E)$. We also denote $((1 \otimes \mathcal{X}(N))(C))$ the ideal in $H_{\text {naive }}^{\bullet}(E)$ generated by the vector subspace $\operatorname{im} T_{3}=\{(1 \otimes X)(C), X \in$ $\mathcal{X}(N)\}$.

Corollary 5.7. The Courant algebroid cohomology of a generalized exact Courant algebroid $E$ with split base $D \cong T L \times N$ is given by

$H_{\mathrm{std}}^{n}(E) \cong \bigoplus_{p+2 q=n} H_{\mathrm{DR}}^{p}(L) \otimes \mathcal{C}^{\infty}(N) /((1 \otimes \mathcal{X}(N))(C)) \otimes_{\mathcal{C}^{\infty}(N)} S^{q}(\operatorname{Ann}(C))$, where $[C]$ is the Ševera class of $E$.

Proof. This is an immediate consequence of Theorem 4.12, Proposition 5.6 and the isomorphism $H_{\text {naive }}^{\bullet}(E) \cong H_{D R}^{\bullet}(L) \otimes \mathcal{C}^{\infty}(N)$.

Remark 5.8. Let $E$ be a generalized exact Courant algebroid with split base $D \cong T L \otimes N$. Assume that the Ševera class of $E$ can be represented by a 3-form $C \in \Omega^{3}(L) \otimes \mathcal{C}^{\infty}(N)$ which is constant as a function of $N$, i.e., $C \in \Omega^{3}(L) \otimes \mathbb{R} \subset \Omega^{3}(L) \otimes \mathcal{C}^{\infty}(N)$. Then, by Proposition 5.6 $T_{3}=0$ and thus, by Corollary 5.7, the cohomology of $E$ is

$$
H_{\mathrm{std}}^{n}(E) \cong \bigoplus_{p+q=n} H_{\mathrm{DR}}^{p}(L) \otimes \mathcal{X}^{q}(N)
$$

An example of such a Courant algebroid is obtained as in Example 5.5 by taking $C=\omega \otimes 1$ for the Ševera 3-form, where $\omega$ is any closed 3-form on $L$.

Example 5.9. Let $G$ be a Lie group with a bi-invariant metric $\langle.,$.$\rangle . Then G$ has a canonical closed 3 -form which is the Cartan 3 -form $C=\left\langle\left[\theta^{\mathrm{L}}, \theta^{\mathrm{L}}\right], \theta^{\mathrm{L}}\right\rangle$ where $\theta^{\mathrm{L}}$ is the left-invariant Maurer-Cartan 1 -form. Note that, by adinvariance of $\langle.,\rangle,$.$C is bi-invariant (hence closed).$

Thus, by Example 5.5, there is a generalized exact Courant algebroid structure on $G \times N$ with Ševera class $[C \otimes f]$ for any manifold $N$ and function $f \in \mathcal{C}^{\infty}(N)$. This example (for $N=\{*\}$ ) was suggested by Alekseev (see also [Roy01, example 5.5]). Explicitly, the Courant algebroid is $E=$ 
$(\mathfrak{g} \oplus \mathfrak{g}) \times N \rightarrow G \times N$. The structure maps are given by

$$
\begin{aligned}
\left\langle X \oplus Y, X^{\prime} \oplus Y^{\prime}\right\rangle & =\left\langle X, X^{\prime}\right\rangle-\left\langle Y, Y^{\prime}\right\rangle, \\
\rho: E \rightarrow T G \boxplus T N:(X \oplus Y, g, n) & \mapsto\left(X^{1}-Y^{\mathrm{r}}\right)(g) \boxplus 0,
\end{aligned}
$$

where the superscript ${ }^{l}$ (resp. ${ }^{\mathrm{r}}$ ) means that an element of the Lie algebra $\mathfrak{g} \cong T_{e} G$ is extended as a left (right) invariant vector field on $G$. The bracket is given, for $(g, n) \in G \times N$ and $X, X^{\prime}, Y, Y^{\prime} \in \mathfrak{g} \subset \Gamma(\mathfrak{g} \times G)$, by

$$
\left[X \oplus X^{\prime}, Y \oplus Y^{\prime}\right]_{(g, n)}=\left(\left[X, X^{\prime}\right] \oplus f(n)\left[Y, Y^{\prime}\right]\right)_{(g, n)} .
$$

Choosing the splitting $\sigma(Z, g, n):=\left(Z \oplus-\operatorname{Ad}_{g} Z, g, n\right)$, one finds that the Cartan 3 -form $C$ is indeed the Severa class of $E$.

Now assume $G$ is a compact simple Lie group, then $C$ spans $H^{3}(G)$. If we take $N=\mathbb{R}$ and $f$ to be constant, then, by Remark 5.8, the cohomology of $E$ is $H_{\text {std }}^{\bullet}(E) \cong H^{\bullet}(G) \otimes \mathcal{X}^{\bullet}(\mathbb{R})$, thus two copies (in different degrees) of the cohomology of $G$. Now take $f \in \mathcal{C}^{\infty}(\mathbb{R})$ to be $f(t)=t$. Then $\operatorname{Ann}(C)$ is trivial and $H_{\text {std }}^{\bullet}(E)=H^{\bullet}(G) /(C)$. Note that $H^{\bullet}(G)$ is the exterior algebra $H^{\bullet}(G) \cong \Lambda^{\bullet}\left(C, x_{2}, \ldots, x_{r}\right)$, thus $H_{\text {std }}^{\bullet}(E) \cong \Lambda^{\bullet}\left(x_{2}, \ldots, x_{r}\right)$ as an algebra, see Remark 4.13.

\section{Appendix A: Graded geometry}

In this appendix we recall some basics of super and graded geometry. For a good and detailed introduction to supermanifolds see, for instance, [Vor91].

Definition A.1. A supermanifold $\mathcal{E}$ of dimension $p \mid q$ is a smooth manifold $M$ of dimension $p$ together with a sheaf, denoted $\mathcal{C}^{\infty}(\mathcal{E})$, of $\mathbb{Z}_{2}$-graded $\mathcal{C}^{\infty}(M)$-algebras locally of the form $\mathcal{C}^{\infty}(U) \otimes_{\mathbb{R}} \Lambda^{\bullet}\left(\mathbb{R}^{q}\right)$.

$\mathcal{C}^{\infty}(\mathcal{E})$ is called the sheaf of super functions on $\mathcal{E}$.

Example A.2. A standard example of a (non-trivial) super manifold is the odd vector bundle $\Pi E$ associated to a smooth vector bundle $E \rightarrow M$. The supermanifold $\Pi E$ is the manifold $M$ together with the structure sheaf $\Gamma_{M}\left(\Lambda^{\bullet} E^{*}\right)$. Locally, $\mathcal{C}^{\infty}(\mathcal{E})$ is generated by $C^{\infty}(U)$ and a local frame $\xi^{1}, \ldots, \xi^{k}$ of $E^{*}$, where $k$ is the rank of $E$.

Example A.2 is fundamental because of the well known

Theorem A.3 (Batchelor). Every supermanifold can be realized as an odd vector bundle.

For a proof of this see, e.g., [Vor91]. In particular, the functor $\Pi$ : Vect $\rightarrow$ SMan (given by Example A.2) from the category of vector bundles to the category of supermanifolds is surjective on the objects. However these two categories are not equivalent, since the category of supermanifolds has more morphisms than the category of vector bundles [Vor91]. 
Vector bundles on supermanifolds are defined analogously to smooth vector bundles, using $\mathbb{Z}_{2}$-graded vector spaces instead of mere non-graded vector spaces. In particular, the tangent bundle of a supermanifold $\mathcal{E}$ is the space of graded derivations of the structure sheaf $\mathcal{C}^{\infty}(\mathcal{E})$.

A.1. Graded manifolds. For graded manifolds we refer to [Vor02, Section 4], [ک̌ev05, Section 2] or [Roy01, Section 2].

The difference between a supermanifold and a graded manifold lies in an additional data for a graded manifold - the Euler vector field - as well as a cover of compatible $\mathbb{Z}$-graded charts. Before recalling the formal definition, let us first consider a fundamental example along the lines of Example A.2.

Example A.4. Let $E \rightarrow M$ be a smooth vector bundle and consider the supermanifold $\mathcal{E}=\Pi T \Pi E$. An Euler vector field $\epsilon$ can be defined, in coordinates induced by charts of $E \rightarrow M$, by the formula

$$
\epsilon=2 \theta^{a} \frac{\partial}{\partial \theta^{a}}+v^{i} \frac{\partial}{\partial v^{i}}+\xi^{a} \frac{\partial}{\partial \xi^{a}}
$$

where $x^{i}, \xi^{a}$ are coordinates on $M$ and the fibers of $E$ and $v^{i}, \theta^{a}$ are their correspondents on $T E$. Note that $\epsilon$ is well defined because for these adapted charts we have the following transition functions:

$$
\begin{aligned}
\widetilde{x^{i}} & =\widetilde{x^{i}}(x), \\
\widetilde{\xi^{a}} & =M_{b}^{\tilde{a}}(x) \xi^{b}, \\
\widetilde{v^{i}} & =\frac{\partial \widetilde{x^{i}}}{\partial x^{j}} v^{j}, \\
\widetilde{\theta^{a}} & =M_{b}^{\tilde{a}} \theta^{b}+M_{b, i}^{\tilde{a}} \xi^{b} v^{i} .
\end{aligned}
$$

Now we restrict $\mathcal{C}^{\infty}(\mathcal{E})$ to functions which are polynomial in $\theta^{a}$ 's. A crucial observation is that the adapted coordinates are eigenfunctions of this Euler vector field $\epsilon$, namely $\epsilon \cdot x^{i}=0, \epsilon \cdot \xi^{a}=\xi^{a}, \epsilon \cdot v^{i}=v^{i}$ and $\epsilon \cdot \theta^{a}=2 \theta^{a}$. Moreover the structure sheaf $\mathcal{C}^{\infty}(\mathcal{E})$ is $\mathbb{Z}$-graded and locally (over such a chart) generated by these coordinates (as smooth functions) in all coordinates of degree 0 , the free exterior algebra in all odd coordinates, and a polynomial algebra in all even coordinates not of degree 0 . Therefore, the eigenvalues of $\epsilon$ are called degrees, and are compatible with the $\mathbb{Z}_{2}$-grading of $\mathcal{C}^{\infty}(\mathcal{E})$, i.e., eigenfunctions for odd eigenvalues are odd functions in the $\mathbb{Z}_{2}$-grading and similarly for even eigenvalues. The supermanifold $\mathcal{E}$ together with this Euler vector field and the charts induced by $E \rightarrow M$ is an example of a graded manifold, denoted $T[1] E[1]$.

Definition A.5 ((Integer) graded manifold). Let $\mathcal{E}$ be a fixed supermanifold with a fixed even vector field $\epsilon$. 
(1) A chart of $\mathcal{E}$ is called $\mathbb{Z}$-graded iff its coordinates are eigenfunctions of $\epsilon$ with integer eigenvalues.

The structure sheaf $\mathcal{C}^{\infty}$ of $\mathbb{Z}$-graded functions over this chart is the $\mathbb{Z}$-graded algebra generated by these coordinates, i.e., smooth functions in the coordinates of degree 0 , the free exterior algebra in the odd coordinates, and the free algebra of symmetric polynomials in the even coordinates not of degree 0 , with the $\mathbb{Z}$-grading given by $\epsilon$.

(2) A $\mathbb{Z}$-graded atlas of $\mathcal{E}$ is an open cover with $\mathbb{Z}$-graded charts such that the transition functions between them preserve the $\mathbb{Z}$-grading and are constituted of $\mathbb{Z}$-graded functions. Especially the number of coordinates in each degree is the same on all charts.

(3) An (integer) graded manifold $\mathcal{E}$ is a supermanifold $\mathcal{E}$ together with a fixed vector field $\epsilon$, called the Euler vector field, and a (maximal) $\mathbb{Z}$-graded atlas.

The graded manifold is said to be non-negatively graded if all coordinates are of non-negative integer degree.

Even though the $\mathbb{Z}_{2}$-grading (even/odd) is not required to be related to the integer grading (by the Euler vector field) in the definition, in many practical examples, the two gradings are compatible, i.e., odd functions with respect to the integer grading are exactly the odd with respect to the $\mathbb{Z}_{2}$-grading. All graded manifolds in this paper fulfill this restriction. Non-negatively graded manifolds for which the two gradings are compatible are also called $N$-manifolds [Roy01].

Example A.6. Given a smooth vector bundle $E \rightarrow M$ and an integer $n \neq 0$, we can form the graded manifold $E[n]$ with the canonical Euler vector field which assigns the degree $n$ to fiber-linear functions as in Example A.4.

There is an obvious notion of vector bundles over graded manifolds similar to the notion of vector bundles over super manifolds. If $\mathcal{E} \rightarrow \mathcal{M}$ is such a vector bundle, one denotes by $\mathcal{E}[n]$, the graded vector bundle obtained by shifting the fiber degrees by $n$.

For instance, any graded manifold $\mathcal{E}$ has a tangent bundle $T \mathcal{E}$ defined as the graded vector bundle of (graded) derivations of $\mathcal{C}^{\infty}(\mathcal{E})$. Similarly, its cotangent bundle $T^{*} \mathcal{E}$ is its $\mathcal{C}^{\infty}(\mathcal{E})$-linear dual.

A sequence of graded manifolds $\mathcal{C} \rightarrow \mathcal{E} \rightarrow \mathcal{F}$ is said to be exact if the sequence of sheaves $\mathcal{C}^{\infty}(\mathcal{C}) \leftarrow \mathcal{C}^{\infty}(\mathcal{E}) \leftarrow \mathcal{C}^{\infty}(\mathcal{F})$ is exact. This provides a bridge between graded geometry and homological algebra.

A.2. Graded Poisson and symplectic manifolds. There is a de Rham differential $d_{\mathcal{E}}: \mathcal{C}^{\infty}(\mathcal{E}) \rightarrow \Omega^{1}(\mathcal{E}):=\Gamma_{\mathcal{E}}\left(T^{*}[1] \mathcal{E}\right)$ for graded manifolds [Vor91, Roy01], generalizing the de Rham differential for smooth (non graded) manifolds, which uniquely extends as a derivation to $\Omega^{\bullet}(\mathcal{E}):=\Gamma_{\mathcal{E}}\left(S^{\bullet}\left(T^{*}[1] \mathcal{E}\right)\right)$. 
Definition A.7. A graded Poisson manifold of degree $d,(\mathcal{E},\{.,\}$.$) is a$ graded manifold $\mathcal{E}$ together with a bracket of degree $d$ on its structure sheaf $\mathcal{C}^{\infty}(\mathcal{E})$ satisfying, for all homogeneous functions $f, g, h \in \mathcal{C}^{\infty}(\mathcal{E})$,

$$
\begin{aligned}
\{g, f\} & =(-1)^{(|f|+d)(|g|+d)+1}\{f, g\}, \\
\{f, g h\} & =\{f, g\} h+(-1)^{(|f|+d)|g|} g\{f, h\}, \\
\{f,\{g, h\}\} & =\{\{f, g\}, h\}+(-1)^{(|f|+d)(|g|+d)}\{g,\{f, h\}\} .
\end{aligned}
$$

A graded presymplectic manifold $(\mathcal{E}, \omega)$ is a graded manifold with a homogeneous $d_{\mathcal{E}}$-closed 2-form $\omega$.

A graded symplectic manifold $(\mathcal{E}, \omega)$ is a graded presymplectic manifold with $\omega$ of total degree $d+2$ such that the map of vector bundles $\omega^{b}$ : $T \mathcal{E} \rightarrow T^{*} \mathcal{E}$ is non-degenerate. It is in particular a graded Poisson manifold of degree $-d$.

Graded Poisson manifolds occur naturally in field theory, more particularly in the context of the AKSZ or BV-formalism. As in the non-graded case, the Poisson bracket is encoded by a homogeneous Poisson bivector $\Pi$, i.e., living in $\Gamma_{\mathcal{E}}\left(S^{2}(T[-1] \mathcal{E})\right)$, satisfying $[\Pi, \Pi]_{S c}=0$, where $[., .]_{S c}$ is the graded analogue of the Schouten bracket.

Example A.8. (1) Let $(E,\langle.,\rangle$.$) be a pseudo-Euclidean vector bundle$ over $M$. Then $E[1]$ has a canonical degree -2 Poisson bracket defined on two sections $\alpha, \beta \in \Gamma_{M}(E[1])$ by $\{\alpha, \beta\}=\langle\alpha, \beta\rangle$. Note that if $f, g \in \mathcal{C}^{\infty}(M) \cong \mathcal{C}^{\infty}(E[1])^{0}$, then $\{f, \alpha\}$ and $\{f, g\}$ are necessarily zero for degree reasons. Note that $(E[1],\{.,\}$.$) is not a graded sym-$ plectic manifold.

Similarly, one defines a degree $-2 n$ Poisson manifold structure on $E[n]$.

(2) The (shifted) cotangent bundles $T^{*}[k] \mathcal{E}$ is a graded symplectic manifold with symplectic form $\omega=d_{T^{*}[k] \mathcal{E}} \theta$ induced by the symplectic potential/Liouville-form $\theta$ similar to the smooth case. Here $\omega$ has total degree $k+2$.

Let $z^{\alpha}$ be coordinates of $\mathcal{E}$ of degree $|\alpha|, p_{\alpha}$ their duals of degree $k-|\alpha|$ and $d:=d_{T^{*}[k] \mathcal{E}}$ the de Rham differential on $T^{*}[k] \mathcal{E}$. The Liouville form reads locally as $\theta:=p_{\alpha} d z^{\alpha}$ of homogeneous degree $k+1$ and the symplectic form reads locally

$$
\omega=d p_{\alpha} d z^{\alpha}
$$

which is obviously closed (under $d$ ) and also non-degenerate. The Poisson bracket on the coordinates reads as

$$
\left\{p_{\alpha}, z^{\beta}\right\}=\delta_{\alpha}^{\beta} \quad\left\{z^{\alpha}, z^{\beta}\right\}=0=\left\{p_{\alpha}, p_{\beta}\right\}
$$

and has degree $-k$. 
Ševera has given the following example of a symplectic manifold which allows the derived bracket construction of Courant algebroids, see Section 2 .

Proposition A.9. Let $(E \rightarrow M,\langle.,\rangle$.$) be a pseudo-Euclidean vector bundle.$ The fiber product $E[1] \times\left(E \oplus E^{*}\right)[1] T^{*}[2] E[1]$ is a graded symplectic manifold where the symplectic form has total degree $2+2$.

Proof. Let us denote $g=\langle.,$.$\rangle the pseudo-metric and g^{\sharp}: E[1] \rightarrow E^{*}[1]$ the isomorphism it induces. Let $i: E[1] \rightarrow\left(E \oplus E^{*}\right)[1]$ be the map $\psi \mapsto \psi \oplus \frac{1}{2} g^{\sharp} \psi$. It is easy to check that $i$ is a morphism of graded Poisson manifolds. Thus the pullback along $i: E[1] \rightarrow\left(E \oplus E^{*}\right)[1]$ of the symplectic form on $T^{*}[2] E[1]$ gives a structure of graded presymplectic manifold on $E[1] \times\left(E \oplus E^{*}\right)[1] T^{*}[2] E[1]$. Let $\omega$ be the closed 2-form. Now you need to check that $\omega$ is non-degenerate. It follows since, in the adapted coordinates from Section 2, $\omega$ and the associated bivector $\Pi$, have the following explicit expression:

$$
\begin{aligned}
& \omega=d p_{i} \wedge d x^{i}+\frac{1}{2} g_{a b} d \xi^{a} d \xi^{b} \\
& \Pi=\frac{\partial}{\partial p_{i}} \wedge \frac{\partial}{\partial x^{i}}+\frac{1}{2} g^{a b} \frac{\partial}{\partial \xi^{a}} \cdot \frac{\partial}{\partial \xi^{b}}
\end{aligned}
$$

\section{References}

[BT82] R. Bott and L. Tu, Differential forms in algebraic topology, Graduate Texts in Mathematics, Vol. 82, Springer-Verlag, New York, 1982, xiv+331pp., ISBN 0387-90613-4.

[CEal] H. Cartan and S. Eilenberg, Homological algebra, Princeton Landmarks in Mathematics, Princeton University Press, Princeton, NJ, 1999, Reprint of the 1956 original, ISBN 0-691-04991-2.

[Cou90] T. Courant, Dirac manifolds, Trans. Amer. Math. Soc. 319 (1990), 631-661, not in arXiv.

[Dor87] I.Y. Dorfman, Dirac structures of integrable evolution equations, Phys. Lett. A, 125 (1987), 240-246.

[Dor93] Dirac stuctures and integrablility of nonliear evolution equations. Nonlinear science: theory and applications. John Wiley \& Sons, Ltd., Chichester, 1993, xii + 176 pp. ISBN 0-471-93893-9.

[Gua04] M. Gualtieri, Generalized complex geometry, Ph.D. thesis, Oxford University 2004, math.DG/0401221.

[Hit03] N. Hitchin, Generalized Calabi-Yau manifolds, Q. J. Math. 54(3), (2003), 281308. math.DG/0209099.

[HP04] C. Hofman and J.-S. Park, BV quantization of topological open membranes, Comm. Math. Phys. 249(2) (2004), 249-271, ISSN 0010-3616. 
[Ike01] N. Ikeda, Deformation of BF-theories, topological open membrane and a generalization of the star deformation, J. High Energy Phys. 07 (2001), 037, hep-th/0105286.

[Ike03] Chern-Simons gauge theory coupled with BF-theory, Int. J. Mod. Phys. A18 (2003), 2689-2702, hep-th/0203043.

[KS96] Y. Kosmann-Schwarzbach, from Poisson algebras to Gerstenhaber algebras, Ann. Inst. Fourier (Grenoble), 46 (1996), 1243-1274.

[LWX97] Z.-J. Liu, A. Weinstein, and P. Xu, Manin triples for Lie bialgebroids, J. Differential Geom. 45/3 (1997), 547-574, math.DG/9508013.

[Mac87] K. Mackenzie, Lie groupoids and Lie algebroids in differential geometry, London Mathematical Society Lecture Note Series, Vol. 124, Cambridge University Press, Cambridge, 1987, xvi+327pp., ISBN 0-521-34882-X.

[McC85] J. McCleary, A user's guide to spectral sequences. Second edition. Cambridge Studies in Advanced Mathematics, Vol. 58. Cambridge University Press, Cambridge, 2001.

[Par01] J.-S. Park, Topological open p-branes, in Symplectic geometry and mirror symmetry (Seoul, 2000), World Sci. Publ., River Edge, NJ, 2001, 311-384, hepth/0012141.

[Roy99] D. Roytenberg, Courant algebroids, derived brackets, and even symplectic supermanifolds, Ph.D. thesis, University of California, Berkley, 1999, math$\mathrm{dg} / 9910078$.

[Roy01] On the structure of graded symplectic supermanifolds and Courant algebroids, in Quantization, Poisson brackets and beyond (Manchester, 2001), Contemp. Math., Vol. 315, Amer. Math. Soc., Providence, RI, 2002, 169-185.

[Roy07] AKSZ-BV formalism and Courant algebroid induced topological field theories, Lett. Math. Phys. 79(2) (2007), 143-159, hep-th/0608150.

[Šev] P. Ševera, Private communication with A. Weinstein and D. Roytenberg.

[Šev99] Letters to A. Weinstein, 1999, http://sophia.dtp.fmph.uniba.sk/ $\sim$ severa/letters/.

[Šev05] P. Ševera, Some title containing the words "homotopy" and "symplectic", e.g. this one, in Travaux mathématiques. Fasc. XVI, Trav. Math., Vol. XVI, Univ. Luxemb., Luxembourg, 2005, 121-137, math.SG/0105080.

[SW99] A. Cannas da Silva and A. Weinstein, Geometric models for noncommutative algebras, Berkeley Mathematics Lecture Notes, Vol. 10, American Mathematical Society, Providence, RI, 1999, xiv+184pp., ISBN 0-8218-0952-0. http://math. berkley.edu/ alanw/.

[ŠW01] P. Ševera, Pavol and A. Weinstein, Poisson geometry with a 3-form background. Noncommutative geometry and string theory (Yokohama, 2001). Progr. Theoret. Phys. Suppl. No. 144 (2001), 145-154.

[SX08] M. Stiénon and P. Xu, Modular classes of loday algebroids, C. R. Acad. Sci. Paris Ser. I 346 (2008), 193-198, math.DG/0803-2047.

[Uch02] K. Uchino, Remarks on the definition of a Courant algebroid, Lett. Math. Phys. 60/2 (2002), 171-175, math.DG/0204010.

[Vor91] T. Voronov, Geometric Integration theory on Supermanifolds, Soviet Scientific Reviews, Section C: Mathematical Physics Reviews, Vol. 9, Harwood Academic Publishers, Chur, 1991, iv+138pp., ISBN 3-7186-5199-8. 
[Vor02] Graded manifolds and Drinfeld doubles for Lie bialgebroids, in Quantization, Poisson brackets and beyond (Manchester, 2001), Contemp. Math., Vol. 315, Amer. Math. Soc., Providence, RI, 2002, 131-168, math.DG/0105237.

UPMC - Université Pierre et Marie Curie Paris 6

Equipe Analyse Algébrique

4, Place Jussieu 75252 PARIs, France

E-mail address: ginot@math.jussieu.fr

Penn State University

109 MC Allister Building

UNIVERSITY PARK, PA 16802

E-mail address: grutzman@math.psu.edu

Received 05/24/2008, accepted 03/05/2009

The authors would like to thank Ping $\mathrm{Xu}$ for various comments on the earlier versions of this paper. 
